\title{
Reflex Modification in the Rat: The Inhibitory Effects of Intensity and Frequency Changes in Steady Tones
}

\author{
Jacquelyn Cranney, Michelle E. Cohen, and Howard S. Hoffman \\ Graduate School of Arts and Sciences, Bryn Mawr College
}

\begin{abstract}
In laboratory rats (as in humans) a low-intensity tone that precedes a highintensity burst of noise by approximately $100 \mathrm{~ms}$ can reduce the amplitude of the startle reaction elicited by the burst of noise. A series of four experiments with rats investigated the relation between the inhibitory effects of tonal frequency change and the length of the silent period (gap) preceding it. The major findings were the following: (a) A gap in an otherwise continuous pure tone inhibited startle when the gap occurred approximately $100 \mathrm{~ms}$ prior to the noise burst. (b) Although an increase in gap duration increased the inhibition afforded by the gap, the maximum inhibition was yielded by gaps of $100 \mathrm{~ms}$ and greater; this maximum was equivalent to the inhibition yielded by the presentation of a postgap tone alone. (c) A shift in tonal frequency across a 10-ms gap yielded more inhibition than did the same gap with no frequency shift; again the shift yielded equivalent inhibition to the presentation of the postgap tone alone. (d) An increase in the frequency shift increased inhibition when the shift occurred across a 10-ms gap, but not when the shift occurred across a 100-ms gap.
\end{abstract}

Previous studies of the acoustic startle reflex have indicated that in the rat (as in the human), response amplitude is determined, in large part, by the sensory events that immediately precede the startle-eliciting event (Hoffman \& Searle, 1965, 1968; Hoffman \& Wible, 1969, 1970). If, for example, a relatively weak stimulus such as a low-intensity tone (a stimulus that does not itself evoke overt startle) precedes an intense burst of noise by approximately $100 \mathrm{~ms}$, the amplitude of the elicited startle reaction is reduced. This inhibitory effect is called reflex modification (Hoffman \& Ison, 1980; Ison \& Hoffman, 1983).

Investigation of the reflex modification afforded by complex acoustic events began with the work of Stitt, Hoffman, Marsh, and Boskoff (1974). They found that if a small change in the intensity or the frequency of

This research was supported by Grant HD 10511 to Howard S. Hoffman from the National Institutes of Health.

We wish to thank Kevin McConkey and two anonymous reviewers for their comments on an early draft of this article.

Jacquelyn Cranney is now at Dartmouth College.

Requests for reprints should be sent to Howard S. Hoffman, Department of Psychology, Bryn Mawr College, Bryn Mawr, Pennsylvania 19010. an ongoing narrow-band noise occurred approximately $100 \mathrm{~ms}$ prior to the presentation of a startle-eliciting stimulus, then the amplitude of the elicited reaction was reduced. More recently, Ison (1982; Ison \& Pinckney, 1983) found that a brief gap in an otherwise continuous noise band functioned in a similar fashion. In addition, Cranney, Hoffman, and Cohen (1984) found that comparable inhibitory effects could be obtained in humans when the ongoing stimulus was a steady tone rather than a band of noise.

The current four experiments were designed to examine in rats the reflex modification afforded by a brief gap and/or a shift in the frequency of an otherwise continuous tone when these events occurred just prior to the presentation of a startle-eliciting burst of noise. Specifically, we investigated the reflex modification afforded by a change in the intensity and/or the frequency of an otherwise steady tone. Information about these effects in rats were important for several reasons: (a) It would provide data relevant to the interpretation of how relatively complex acoustic events are processed. (b) It would provide a basis for future neurophysiological investigations. (c) It would provide a basis for future work in areas such as animal psychophysics and behavioral toxicology be- 
cause reflex modification procedures have proved to be useful in assessing the specific sensory and/or behavioral deficits engendered by toxic substances (e.g., Kellogg, Ison, \& Miller, 1983; Young \& Fechter, 1983).

\section{Experiment 1}

Experiment 1 was designed to determine how the amount of reflex modification afforded by a gap in an otherwise steady tone varied as a function of the duration of the gap. The experiment was designed to extend the work of Ison (1982), who found that inhibition increased when the duration of a gap in a noise band increased. We expected that a similar phenomenon would occur with pure tones.

\section{Method}

Subjects. The subjects were 9 experimentally naive male albino rats obtained from the Charles River Breeding Laboratories. They were approximately 90 days old at the time of testing.

Apparatus. The test apparatus was housed in an Industrial Acoustics Corporation sound-attenuated chamber fitted with sound-absorbing material to reduce acoustic reflections. The animal test chamber has been described in detail by Hoffman and Wible (1969). It consisted of a small cage $(20.3 \mathrm{~cm} \times 12.7 \mathrm{~cm} \times 15.2$ $\mathrm{cm}$ ) constructed of stainless-steel rods suspended from a rigid superstructure by four thin pieces of epoxy and fiberglass laminate. An aluminum rod with a magnet on its distal end was mounted on top of the cage so that the magnet rode inside a coil that was fastened to the superstructure. With this arrangement, any sudden movement of the cage, for example, during a startle reflex, would cause the magnet to move in the coil and generate an electrical current. This current was filtered to eliminate stimulus artifacts and amplified before being passed to a Coulbourn peak detector (Module S76-31), which assessed the response during the $150-\mathrm{ms}$ period that began with the onset of the startle-eliciting stimulus. The output of the peak detector was digitized and strobed to a Radio Shack TRS-80 microcomputer.

The startle-eliciting stimulus, as well as the acoustic stimuli that preceded it, was shaped and timed by Coulbourn programming modules interfaced with the microcomputer. The outputs of the stimulus-generating modules were amplified by separate channels of a Crown DC 300 audio power amplifier. The startle-eliciting stimulus was presented through a semiconductor gate (see Stitt, Hoffman, \& Marsh, 1976, for a description) to a speaker consisting of an Altec $230 \mathrm{C}$ midrange driver with a $60-\mathrm{cm}$ exponential horn mounted inside the Industrial Acoustics chamber a few inches from the test cage. The acoustic prestimuli were presented through a Realistic Liquid Cooled Super Tweeter that was mounted on the side of the Industrial Acoustics chamber opposite the
Altec driver. The intensities of the acoustic prestimuli were assessed using the Flat scale setting of a General Radio 1561-A sound level meter. All intensities reported here are sound pressure level (SPL re $20 \mu \mathrm{Nm}^{2}$ ).

Stimulus conditions. The acoustic prestimuli were 5 $\mathrm{kHz}$ pure tones with rise-fall times of $5 \mathrm{~ms}$. The startleeliciting stimulus was a $20-\mathrm{ms}$ burst of $120 \mathrm{~dB}$ (SPL) noise with a $0.1-\mathrm{ms}$ rise-fall time. Four basic stimulus configurations were employed in this experiment (see Figure 1).

1. A startle-eliciting stimulus presented alone; a burst of white noise presented with no prior acoustic stimulation (no prestimulus).

2. A 10-s tone, the offset of which coincided with the onset of the startle-eliciting stimulus (zero gap).

3. A startle-eliciting stimulus presented in the presence of a 10-s tone that contained a brief intensity decrement (gap). The onset of the gap began at varying periods $(80,85,95 ; 115,155,395$, and $1,355 \mathrm{~ms})$ and always ended $75 \mathrm{~ms}$ prior to the startle-eliciting stimulus. Thus, there were seven distinct conditions with varying durations of gap $(5,10,20,40,80,320$, and 1,280 $\mathrm{ms}$; the 5-, 10-, and 40-ms gaps are illustrated in Figure 1). Because these gaps included the $5-\mathrm{ms}$ fall and the 5-ms rise time of the tone, the 5-, and $10-\mathrm{ms}$ conditions did not contain a silent period, just an intensity decrement.

4. An 80-ms tone, the offset of which coincided with the onset of the startle-eliciting stimulus (onset).

In all stimulus configurations involving an acoustic

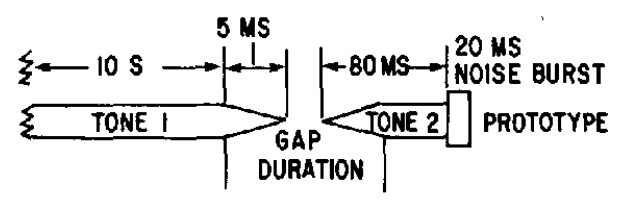

NO PRESTIMULUS
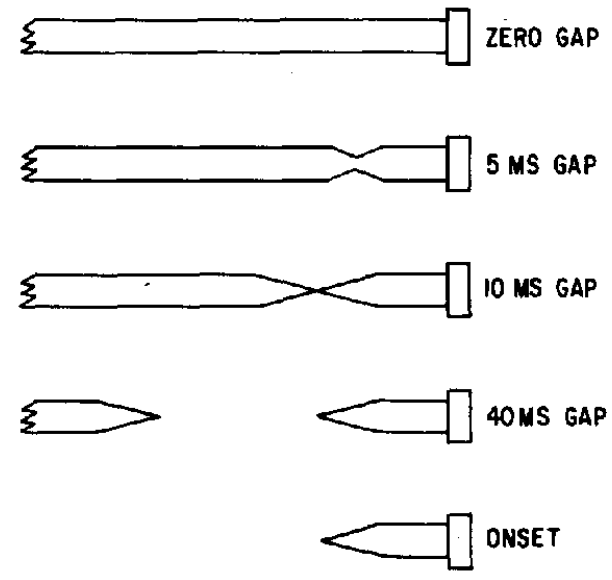

Figure 1. Representative conditions used in Experiment 1 (not to scale). (The top configuration is the prototype for the stimulus conditions.) 
prestimulus, the intensity of the prestimulus was always $70 \mathrm{~dB}$ (SPL), and the onset of the startle-eliciting stimulus always coincided with the beginning of the 5 -ms fall of the acoustic prestimulus. There was a total of 10 kinds of trials, each of which ended with a startle-eliciting burst of noise.

Procedure. Fach subject received 10 blocks of 10 trials, for a total of 100 trials. Within a block of trials, the stimulus conditions were presented randomly, and there was an intertrial interval of $30 \pm 5 \mathrm{~s}$. Each subject was tested for one session, which lasted approximately $70 \mathrm{~min}$; this included an initial 5-min adaptation period.

\section{Results and Discussion}

The basic datum in these experiments was the mean amplitude of startle across trials for each condition for each subject. Figure 2 presents the mean amplitude of startle responses elicited by the 120-dB (SPL) noise burst alone (the horizontal line), as well as the mean amplitude of responses elicited by the same noise burst when it was preceded by a tone with a given gap. It is apparent that when gaps were inserted in the tones, response amplitude declined as a negatively accelerated function of the size of the gap; that is, longer gaps afforded greater inhibition (i.e., decreased the amplitude of startle responding). A repeated measures analysis of variance confirmed that these differences were reliable, $F(9,72)=11.01, p<.05$. A Newman-Keuls analysis $(p<.05)$ revealed that (a) the response amplitudes of the no-prestimulus and zero-gap conditions were not significantly different; (b) conditions with gap durations of $80 \mathrm{~ms}$ and greater produced significantly lower response amplitudes than did the zero-gap condition; (c) conditions with gap durations of $320 \mathrm{~ms}$ and greater produced significantly lower response amplitudes than did the no-prestimulus condition; and (d) the response amplitudes of conditions with gap durations of $320 \mathrm{~ms}$ and greater were not significantly different from that produced by the infinite-gap condition. The 80 ms gap condition, then, inhibited the startle responding with reference to the zero-gap condition, but not with reference to the noprestimulus condition.

The results of Experiment 1 indicate that a gap of sufficient duration in a pure tone significantly reduces the amplitude of the startle response; moreover, the gap function in this experiment is similar to Ison's (1982) gap duration function. This suggests that despite the differences in stimuli employed in this and in Ison's (1982) study, enough similarities are evident in the data to conclude that the inhibitory effect of a gap in an otherwise continuous acoustic prestimulus is a robust phenomenon.

A further point concerning the gap duration function is that maximum inhibition produced by a long gap (i.e., $320 \mathrm{~ms}$ and greater) was equivalent to the inhibition produced by the postgap tone alone (onset condition). In this sense, the onset condition appeared to reflect a gap of infinite duration. Thus, it appears that as the duration of the gap in an otherwise continuous tone increases, it more closely approximates the tonal-onset condition and produces greater inhibition.

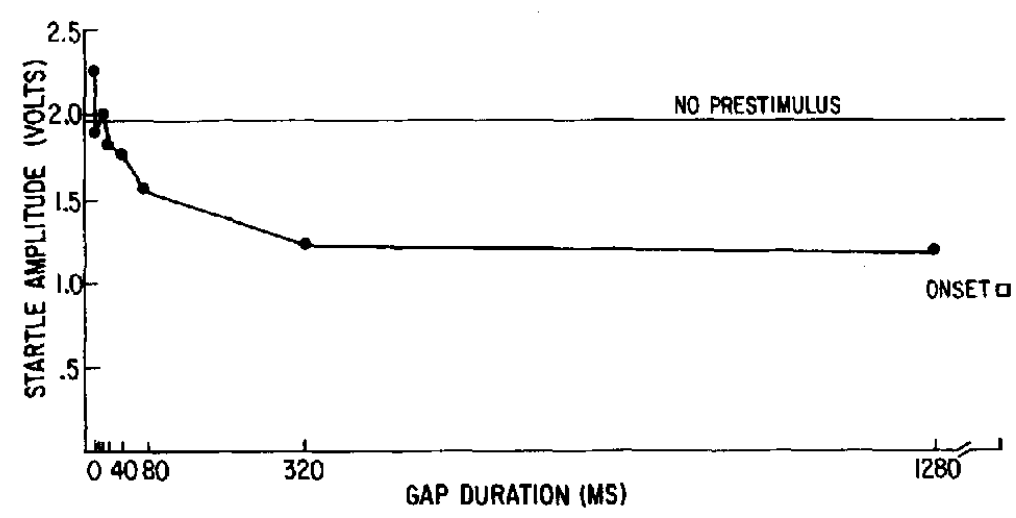

Figure 2. Mean amplitude of startle response (across subjects) as a function of gap duration. (The horizontal line indicates the mean amplitude of startle response elicited by the noise burst without prior acoustic stimulation.) 
This aspect of the results suggests that sensory adaptation may occur during the 10$s$ period of constant tonal prestimulation prior to the gap. With short gaps there may be insufficient time for complete sensoryneural recovery so that during the postgap tone there is less than normal activation of central auditory systems. With longer gaps, however, there may be sufficient time for complete recovery so that during the postgap tone there is as much activation of central auditory systems as there is in the onset condition (where there has been no prior tonal stimulation). Thus, the period between 80 and 320 ms may be critical for recovery from sensory-neural adaptation. To date, however, there have been no sensory adaptation studies employing the stimulus parameters characteristic of the startle reflex experiment (Ison, 1982).

\section{Experiment 2}

Experiment 2 examined whether a shift in frequency during a gap in an otherwise continuous pure tone would lead to any further reflex inhibition than the amount of inhibition due to an intensity decrement with no frequency shift. Previous work (Marsh, Hoffman, Stitt, \& Schwartz, 1975; Stitt et al., 1974) indicated that shifts between high- and lowfrequency bands of noise could inhibit a startle reflex. The question addressed in this experiment was whether similar effects could be obtained when the basic acoustic prestimulus was a pure tone rather than narrowband-noise and when the frequency shift occurred in the context of an intensity decrement (gap) in the prestimulus. Further, the experiment compared the inhibition afforded by frequency shifts during a gap with that afforded by tonal offsets and onsets.

\section{Method}

Subjects and apparatus. The subjects were 14 male albino rats obtained from the Charles River Breeding Laboratories. They were approximately 120 days old at the time of testing. The apparatus was that employed in Experiment 1.

Stimulus conditions. The acoustic prestimuli were 70-dB (SPL), 5- and 10-kHz tones, with a rise-fall time of $5 \mathrm{~ms}$. The startle-eliciting stimulus was a 20 -ms burst of $120-\mathrm{dB}$ (SPL) white noise with a $0.1-\mathrm{ms}$ rise-fall time. Six basic stimulus configurations were employed in this experiment (see Figure 3):
1. A startle-eliciting stimulus presented alone; a burst of white noise presented with no prior acoustic stimulation (no prestimulus).

2. A $10-\mathrm{s}, 5$ - or $10-\mathrm{kHz}$ tone, the offset of which coincided with the onset of the startle-eliciting stimulus (zero gap).

3. A $10-\mathrm{s}, 5-$ or $10-\mathrm{kHz}$ tone with a $10-\mathrm{ms}$ intensity decrement (gap), which ended $75 \mathrm{~ms}$ prior to the startle-eliciting stimulus (gap-no shift).

4. A 10-s, 5- or $10-\mathrm{kHz}$ tone with a 10-ms gap, which ended $75 \mathrm{~ms}$ prior to the startle-eliciting stimulus, with a shift in frequency across the gap (shift).

5. An $80-\mathrm{ms}, 5-$ or $10-\mathrm{kHz}$ tone, the offset of which coincided with the onset of the startle-eliciting stimulus (onset).

6. A 10-s, 5- or 10-kHz tone, the offset of which occurred $154 \mathrm{~ms}$ prior to the startle-eliciting stimulus (offset).

In all stimulus configurations involving an acoustic prestimulus, except the offset condition, the onset of the startle-eliciting stimulus coincided with the 5-ms fall of the acoustic prestimulus. Because tone conditions were counterbalanced for frequency $(5 \mathrm{kHz}$ and $10 \mathrm{kHz}$ ), there was a total of 11 stimulus conditions.

Procedure. Each subject received 10 blocks of 11 trials, for a total of 110 trials. Within a block of trials, the stimulus conditions were presented randomly, and there was an intertrial interval of $30 \pm 5 \mathrm{~s}$. Each subject was tested for one session, which lasted approximately $75 \mathrm{~min}$; this included an initial 5 -min adaptation period.

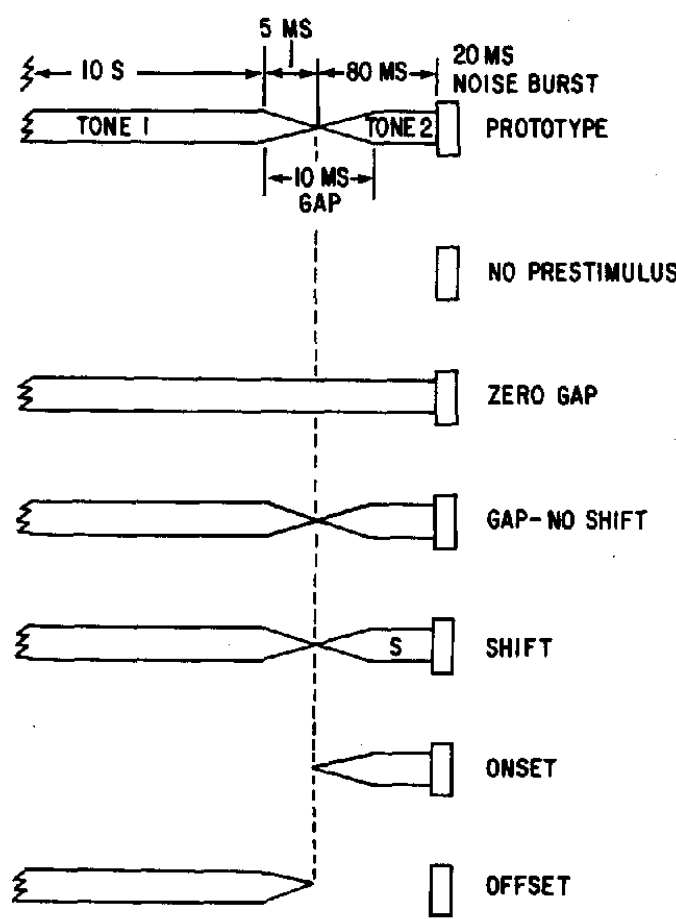

Figure 3. The set of conditions used in Experiment 2 (not to scale). (The top configuration is the prototype for the stimulus conditions. $S$ signifies a shift in frequency.) 


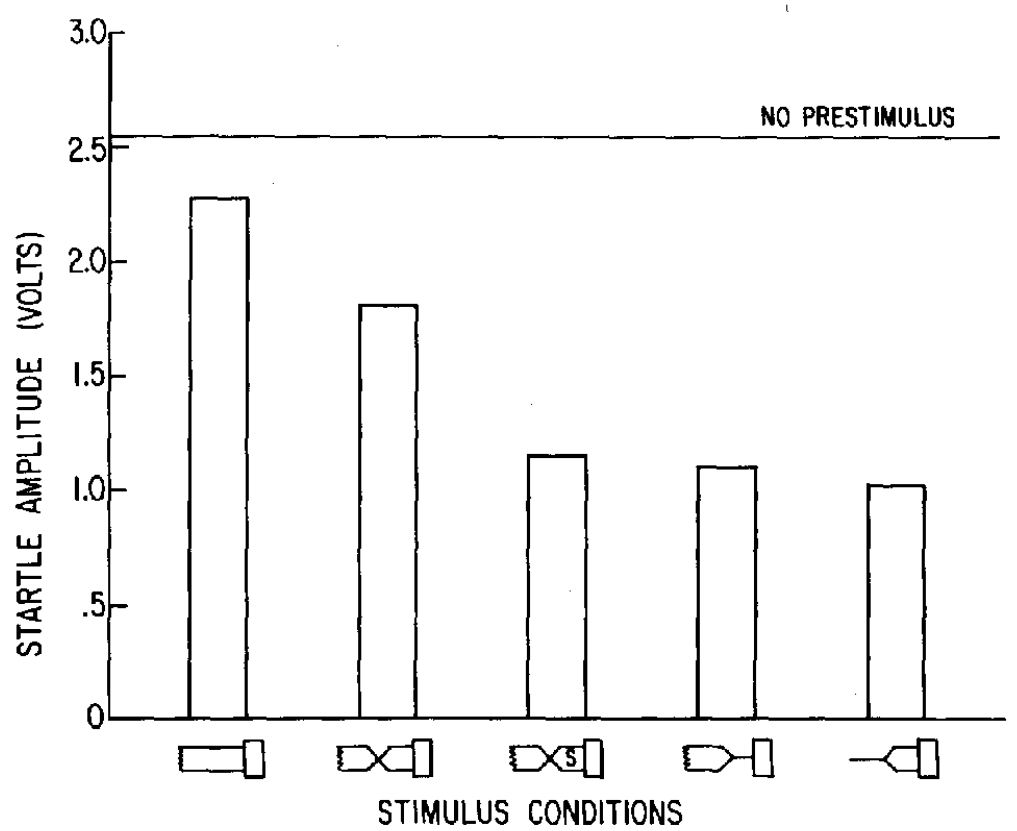

Figure 4. Mean amplitude of startle response (across subjects) for each of the stimulus configurations. (The latter are illustrated schematically below the horizontal axis of the figure. The horizontal line indicates the mean amplitude of startle response elicited by the noise burst without prior acoustic stimulation).

\section{Results and Discussion}

A two-way repeated measures analysis of variance (Prestimulus Condition $\times$ Tonal Frequency) yielded a significant main effect for prestimulus condition, $F(4,117)=45.04$, $p<.05$, but no significant main effect for tonal frequency and no significant interaction effect. Thus, the baseline tonal frequency did not differentially influence startle responding.

Figure 4 presents the mean amplitude of the startle (across subjects) for each of the prestimulus conditions collapsed across baseline frequency. Compared to the no-prestimulus condition, the gap-no shift condition produced smaller responses, but the frequency shift, offset, and onset conditions produced even smaller responses. The reliability of these findings was supported by a NewmanKeuls analysis $(p<.05)$. Responding in the no-prestimulus and zero-gap conditions was equivalent and significantly greater than in all other conditions. There were no significant differences among the frequency shift, offset, and onset conditions, but each of these three conditions produced more inhibition than did the gap-no shift condition.

The results of Experiment 2 indicate that a frequency shift across a gap produced more inhibition than did a gap alone, but no more inhibition than did a simple onset or offset. These findings are similar to those reported with the onsets, offsets, and frequency shifts in otherwise continuous noise bands (Stitt et al., 1974). In addition, the effects of the 5and $10-\mathrm{kHz}$ frequencies in the present experiment corroborate other reports of no differential effects of baseline frequency in noise bands (Stitt et al., 1974) and in pure tones (Cranney et al., 1984), and they are consistent with psychophysical data that rats are equally sensitive to $5-$ and $10-\mathrm{kHz}$ tones (Stebbins, 1970).

\section{Experiment 3}

Experiment 1 revealed that inhibition of the startle reflex due to a gap is optimal with gap durations greater than $80 \mathrm{~ms}$. Experiment 2 revealed that a frequency shift across a short (10-ms) gap produced more inhibition than did a gap alone. Experiment 3 was designed to determine the manner in which these two factors, gap duration and amount of frequency shift, interact. 


\section{Method}

Subjects and apparatus. The subjects were 11 experimentally naive male albino rats obtained from the Charles River Breeding Laboratories. They were approximately 90 days old at the time of testing. The apparatus was that used in Experiment 1.

Stimulus conditions. All tones were 70-dB (SPL) with a 5 -ms rise-fall time. The startle-eliciting stimulus was a $20-\mathrm{ms}$ burst of $120-\mathrm{dB}$ (SPL) white noise with a $0.1-\mathrm{ms}$ rise-fall time. Five basic stimulus configurations were employed in this experiment:

1. A startle-eliciting stimulus alone; a burst of white noise presented with no prior acoustic stimulation (no prestimulus).

2. A $10-\mathrm{s}, 7.5-\mathrm{kHz}$ tone, the offset of which coincided with the onset of the startle-eliciting stimulus (zero gap).

3. An $80-\mathrm{ms}, 5-\mathrm{kHz}$ tone, the offset of which coincided with the onset of the startle-eliciting stimulus (onset).

4. A $10-\mathrm{s}, 5-\mathrm{kHz}$ tone with a $10-\mathrm{ms}$ or $110-\mathrm{ms}$ gap, which ended $75 \mathrm{~ms}$ prior to the startle-eliciting stimulus (gap-no shift).

5. A 10-s tone with a $10-\mathrm{ms}$ or 110-ms gap, which ended $75 \mathrm{~ms}$ prior to the startle-eliciting stimulus, with a downward shift in frequency across the gap (shifts). The frequency shifts were $6.25 \mathrm{kHz}$ to $5 \mathrm{kHz}$ (1.25$\mathrm{kHz}$ shift), $7.5 \mathrm{kHz}$ to $5 \mathrm{kHz}(2.5-\mathrm{kHz}$ shift), and 10 $\mathrm{kHz}$ to $5 \mathrm{kHz}$ (5-kHz shift).

In the stimulus configurations involving an acoustic prestimulus, the onset of the startle-eliciting stimulus coincided with the 5-ms fall of the acoustic prestimulus. There was a total of 11 stimulus conditions.

Procedure. Each subject received 10 blocks of 11 trials, for a total of 110 trials. Within a block of trials, the stimulus conditions were presented randomly, and there was an intertrial interval of $30 \pm 5 \mathrm{~s}$. Each subject was tested for one session, which lasted approximately $75 \mathrm{~min}$; this included an initial 5 -min adaptation period.

\section{Results and Discussion}

Figure 5 presents the mean startle amplitude, averaged across subjects for each of the 11 stimulus conditions. With a short gap, the shift in frequency produced a greater decrease in the startle amplitude than did the gap without the shift. With a long gap, the shift and no-shift conditions produced the same decrement in startle amplitude.

A two-way repeated measures analysis of variance indicated significant main effects for gap duration, $F(1,70)=16.46, p<.05$, and for amount of frequency shift, $F(3,70)=$ $6.66, p<.05$, and a significant interaction effect, $F(3,70)=2.91, p<.05$. Analyses of the simple main effects were performed to assess the details of this interaction. The major findings were a significant effect for amount of frequency shift in the short (10 ms) gap condition, $F(3,70)=8.97, p<.05$, but not in the long $(110 \mathrm{~ms})$ gap condition.

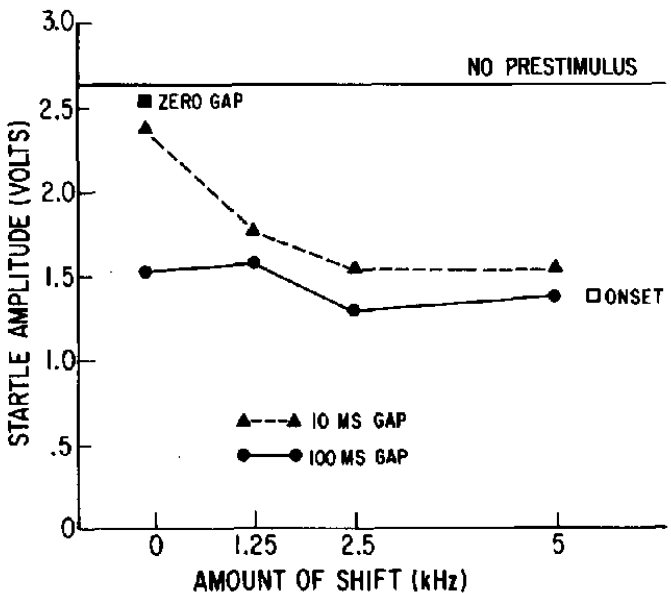

Figure 5. Mean amplitude of startle response (across subjects) as a function of the amount of frequency shift and the gap duration. (The horizontal line indicates the mean amplitude of startle response elicited by the noise burst without prior acoustic stimulation.)

The results of Experiment 3 indicate that a frequency shift added to the amount of inhibition only when the gap duration was relatively short ( $10 \mathrm{~ms})$. A longer gap duration (100 ms) appeared to produce maximum inhibition, and the frequency shift did not have any effect. This suggests that a frequency shift may overcome some of the sensoryneural adaptation effects postulated to be present in the postgap tone of the short gap condition. Such a frequency shift, however, will have no effect on the long gap condition, because once the effects of prior stimulation have decayed, the frequency shift will be no more effective than the resumption of the original frequency.

\section{Experiment 4}

Experiment 3 revealed that a frequency shift of at least $1.25 \mathrm{kHz}$ across a 10 -ms gap resulted in greater inhibition than did a 10ms gap with no frequency shift. Experiment 4 was designed to determine the impact on startle response of frequency shifts of less than $1.25 \mathrm{kHz}$.

\section{Method}

Subjects and apparatus. The subjects were 16 male albino rats obtained from the Charles River Breeding Laboratories. They were approximately 100 days old at the time of testing. The apparatus was that used in Experiment 1. 
Stimulus conditions. All tones were $70 \mathrm{~dB}$ (SPL) with a 5-ms rise-fall time. The startle-eliciting stimulus was a $20-\mathrm{ms}$ burst of $120-\mathrm{dB}$ (SPL) white noise with a $0.1-\mathrm{ms}$ rise-fall time. Four basic stimulus configurations were employed in this experiment:

1. A startle-eliciting stimulus alone; a burst of white noise with no prior acoustic stimulation (no prestimulus).

2. An $80-\mathrm{ms}, 5-\mathrm{kHz}$ tone, the offset of which coincided with the onset of the startle-eliciting stimulus (onset).

3. A $10-\mathrm{s}, 5-\mathrm{kHz}$ tone with a 10-ms gap, which ended $75 \mathrm{~ms}$ prior to the startle-eliciting stimulus (gap-no shift).

4. A 10-s tone with a 10-ms gap, which ended 75 . ms prior to the startle-eliciting stimulus, with a downward shift across the gap (shifts). The frequency shifts were $5.075 \mathrm{kHz}$ to $5 \mathrm{kHz}(0.075-\mathrm{kHz}$ shift $), 5.15 \mathrm{kHz}$ to 5 $\mathrm{kHz}(0.15-\mathrm{kHz}$ shift $), 5.3 \mathrm{kHz}$ to $5 \mathrm{kHz}(0.3-\mathrm{kHz}$ shift), $5.65 \mathrm{kHz}$ to $5 \mathrm{kHz}(0.65-\mathrm{kHz}$ shift), and 6.25 $\mathrm{kHz}$ to $5 \mathrm{kHz}(1.25-\mathrm{kHz}$ shift $)$.

In all of the stimulus configurations involving a prestimulus, the onset of the startle-eliciting stimulus coincided with the 5-ms fall of the acoustic prestimulus. In this experiment, a zero-gap condition was not included because the previous experiments had found no differences between the zero-gap and the no-prestimulus conditions. There was a total of eight stimulus conditions.

Procedure. Each subject received 10 blocks of 8 trials, for a total of 80 trials. Within a block of trials, the stimulus conditions were presented randomly, and there was an intertrial interval of $30 \pm 5 \mathrm{~s}$. Each subject was tested for one session, which lasted approximately 60 min; this included an initial 5-min adaptation period.

\section{Results and Discussion}

Figure 6 presents the mean startle amplitude, averaged across subjects, for all eight conditions. The figure shows that a shift in frequency as small as $0.075 \mathrm{kHz}(5.075 \mathrm{kHz}$ to $5 \mathrm{kHz}$ ) results in a decrease in startle amplitude from the no-shift condition $(5 \mathrm{kHz}$ to $5 \mathrm{kHz}$ ). A one-way repeated measures analysis of variance indicated that these differences were reliable, $F(7,105)=20.05, p<$ .05 . A Newman-Keuls analysis $(p<.05)$ revealed that the no-prestimulus condition was significantly greater than all of the other conditions; this indicated that even a short gap with no frequency shift resulted in a decrease in the startle amplitude. However, the no-shift condition resulted in a significantly greater startle amplitude than did all of the frequency shift conditions and the onset condition. That is, a frequency shift as small as $0.075 \mathrm{kHz}$ across a gap in acoustic stimulation produced a greater reduction in startle amplitude than that produced by a condition in which there was a gap but no frequency shift. This aspect of the data implies

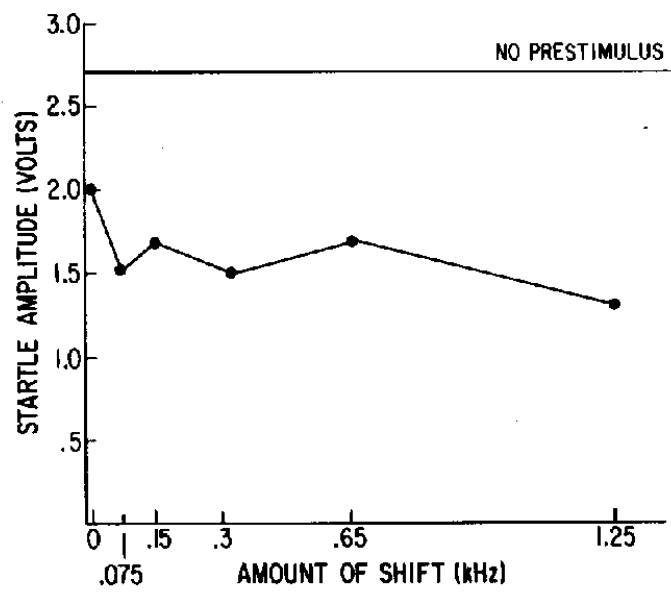

Figure 6. Mean amplitude of startle response (across subjects) as a function of the amount of frequency shift. (The horizontal line in the figure indicates the mean amplitude of startle response elicited by the noise burst without prior or concurrent acoustic stimulation.)

that the threshold for the frequency shift advantage, with respect to a downward shift to $5 \mathrm{kHz}$, must be less than $0.075 \mathrm{kHz}$.

\section{General Discussion}

This series of experiments revealed that a gap in a pure tone, like a gap in a noise band (Ison, 1982), results in a reduction of startle amplitude if presented at an appropriate interval prior to the startle-eliciting stimulus. In addition, the work revealed the following results: (a) Increased gap duration increased the amount of inhibition; gaps of $100 \mathrm{~ms}$ and greater produced inhibition equivalent to that of the postgap tone alone. (b) A frequency shift as small as $0.075 \mathrm{kHz}$ (to 5 $\mathrm{kHz}$ ) across a 10-ms gap produced more inhibition than did the gap with no frequency shift. (c) Frequency shifts did not increase the amount of inhibition afforded by the 100 ms gap. These findings suggest that approximately $100 \mathrm{~ms}$ are required for the effects of prestimulation to decay and that once the effects of prior stimulation have decayed, the frequency shift is no longer effective. That is, with the longer gap the shift is no more effective than with resumption of the pregap tone frequency.

The shortest gap duration that produced inhibition was $20 \mathrm{~ms}$ in Experiment 1 and $10 \mathrm{~ms}$ in Experiments 2 and 4. This suggests 
that the gap duration threshold for reflex inhibition lies between 10 and $20 \mathrm{~ms}$. A 15ms gap includes a 5-ms silent period, which is comparable to Ison's (1982) estimated threshold of $3.5 \mathrm{~ms}$ for the inhibitory effect of a gap in a noise band.

The threshold for the frequency shift advantage in inhibition for rats was determined to be below $0.075 \mathrm{kHz}$; this corresponds to the psychophysically determined threshold for frequency shift detection. In contrast, the human data indicate that the threshold for the frequency shift advantage in inhibition is greater (i.e., the method is less sensitive) than the psychophysically determined threshold for frequency shift detection (Cranney et al., 1984). This finding suggests that the reflex modification procedure taps a low level of auditory processing in humans.

In the present experiments, the zero-gap condition afforded an equivalent amount of inhibition to the no-prestimulus condition. This finding is inconsistent with previous reports that a constant, uninterrupted tonal prestimulus or background noise increases startle responding in the rat (Hoffman \& Searle, 1968; Stitt et al., 1974). One possible reason for this discrepancy is that the current study employed a relatively short, pure tone prestimulus, which may not have been sufficient to produce facilitation.

A major conclusion of the current study is that the amount of inhibition afforded by a complex acoustic event is not merely an additive function of the inhibitory effects of the different elements that make up the event. For instance, a tonal onset is just as inhibitory as a frequency shift across a gap (which includes a tonal onset, a tonal offset, and a frequency shift). One possible explanation of this is in terms of sensory-neural adaptation and the amount of change that occurs in the overall pattern of firing elicited by the acoustic events within the critical period prior to a startle-eliciting stimulus. Although the present findings are consistent with this suggestion, further research that employs appropriate electrophysiological techniques is required to test it directly. Overall, the current study has extended knowledge of the reflex inhibitory effects of complex acoustic events and has provided some directions for future research into the mechanisms underlying reflex inhibition.

\section{References}

Cranney, J., Hoffman, H. S., \& Cohen, M. E. (1984). Tonal frequency shifts and gaps in acoustic stimulation as reflex inhibitory events. Perception \& Psychophysics, $35,165-172$.

Hoffman, H. S., \& Ison, J. R. (1980). Reflex modification in the domain of startle: 1 . Some empirical findings and their implications for how the nervous system processes sensory input. Psychological Review, 87, 175-189.

Hoffman, H. S., \& Searle, J. L. (1965). Acoustic variables in the modification of startle reaction in the rat. Journal of Comparative and Physiological Psychology. $60,53-58$.

Hoffman, H. S., \& Searle, J. (1968). Acoustic and temporal factors in the evocation of startle. Journal of the Acoustical Society of America. 43, 269-282.

Hoffman, H. S., \& Wible, B. (1969). Temporal parameters in startle facilitation by steady background stimulus. Journal of the Acoustical Society of America, 45, 712.

Hoffman, H. S., \& Wible, B. L. (1970). Role of weak stimulus in acoustic startle. Journal of the Acoustical Society of America, 47, 489-497.

Ison, J. R. (1982). Temporal acuity in auditory function in the rat: Reflex inhibition by brief gaps in noise. Journal of Comparative and Physiological Psychology. 96, 945-954.

Ison, J. R., \& Hoffman, H. S. (1983). Reflex modification in the domain of startle: II. The anomalous history of a robust and ubiquitous phenomenon. Psychological Bulletin, 94, 3-17.

Json, J. R., \& Pinckney, L. A. (1983). Reflex inhibition in humans: Sensitivity to brief silent periods in white noise. Perception \& Psychophysics, 34, 84-88.

Kellogg, C., Ison, J. R., \& Miller, R. K. (1983). Auditory temporal resolution in rats: Effect of prenatal diazepam exposure. Psychopharmacologia, 79, 332-337.

Marsh, R., Hoffman, H. S., Stitt, C. L., \& Schwartz, G. M. (1975). The role of small changes in the acoustic environment in modifying the startle reflex. Journal of Experimental Psychology: Animal Behavior Processes, I, 235-244.

Stebbins, W. C. (Ed.). (1970). Animal psychophysics: The design and conduct of sensory experiments. New York: Appleton-Century-Crofts.

Stitt, C. L., Hoffman, H. S., \& Marsh, R. R. (1976). Interaction versus independence of startle-modification processes in the rat. Journal of Experimental Psychology: Animal Behavior Processes, 2, 260-265.

Stitt, C. L.. Hoffman, H. S., Marsh, R,, \& Boskoff, K. J. (1974). Modification of the rat's startle reaction by an antecedent change in the acoustic environment. Journal of Comparative and Physiological Psychology, 86, 826836.

Young, J. S., \& Fechter, L. D. (1983). Reflex inhibition procedures for animal audiometry: A technique for assessing ototoxicology. Jourmal of the Acoustical Society of America, 73, 1686-1693.

Received January 16,1983

Revision received May 1, 1984 